\section{Are alcohol containing mouthwashes safe?}

\author{
C.W. de A. Werner ${ }^{1}$ and R. A. Seymour ${ }^{2}$
}

IN BRIEF

- Dentists need to be aware that there is a hypothetical risk for the development of oral cancer from repeated use of alcohol containing mouthwashes.

- The existing evidence to support or refute such an association has been the subject of two recent conflicting reviews.

- This paper critically evaluates the two reviews and further explores the data on the efficacy of the addition of alcohol to mouthwashes.

Alcohol (ethanol) is a constituent of many proprietary mouthwashes. Some studies have shown that regular use of such mouthwashes can increase the risk of developing oral cancer. Recently, the evidence has been reviewed by two separate authors. The conclusions from these reviews are conflicting. In this paper, we reconsider the epidemiological evidence linking alcohol containing mouthwashes with an increased risk of oral cancer. The evidence is considered in term of sample size, strength of association, confounding variables and data collection. In addition, clinical studies comparing alcohol versus non-alcohol mouthwashes are evaluated. The evidence suggests that the alcohol component of mouthwashes affords little additional benefit to the other active ingredients in terms of plaque and gingivitis control. In view of this outcome and the hypothetical risk of oral cancer, it would seem prudent that members of the dental team advise their patients accordingly.

\section{INTRODUCTION}

Many proprietary mouthwashes contain alcohol (ethanol) and in some, the concentration of ethanol can be as high as $26 \%{ }^{1}$ Two recent reviews ${ }^{2,3}$ have considered whether alcohol containing mouthwashes increase the risk of the development of oral cancer. The conclusions from these reviews are conflicting, with one stating 'there is now sufficient evidence to accept the proposition that alcohol containing mouthwashes contribute to the increase of the development of oral cancer', ${ }^{2}$ while the other states that 'critical review of the published data revealed that a link between mouthwash use, specifically alcohol containing mouthwash and oral cancers, is not supported by epidemiological evidence. ${ }^{3}$

The reviews were published in 2008 and 2009 and for the most part have quoted the same evidence to support their conclusion. In this paper we shall look critically at both

\footnotetext{
I*Clinical Lecturer in Dental Public Health, ${ }^{2}$ Professor of Restorative Dentistry, School of Dental Sciences, Newcastle University, Framlington Place, Newcastle upon Tyne, NE2 4BW

${ }^{*}$ Correspondence to: Dr Carlos Werner

Email: carlos.werner@ncl.ac.uk
}

\section{Online article number E19}

Refereed Paper - accepted 29 October 2009

DOI: 10.1038/sj.bdj.2009.1014

${ }^{\circledR}$ British Dental Journal 2009; 207: E19 reviews and the interpretation of the evidence and hopefully provide readers with an opinion on the safety or otherwise of alcohol containing mouthwashes.

\section{EPIDEMIOLOGICAL EVIDENCE}

Epidemiological studies are complex and aspects such as sample size, strength of association, confounding variables and data collection bias can be rightfully challenged. By contrast, arguments can also be made for the exploitation of specific aspects of interest (reporting bias). This is obviously a controversial topic which can lead to different courses of clinical action. Dentistry often deals with such issues and dentists have to make ethical decisions on clinical protocols and material based on less than ideal types of scientific evidence.

Our aim is to guide the reader through a clear pathway which provides relevant information for reflection and leads to an informed decision. Scientific evidence will be explored in light of the most recent reviews so that we can empower all members of the dental team with the best clinical advice on the use of mouthwashes.

\section{Centre for Evidence Based Dentistry (CEBD)}

The CEBD suggests the following stepwise approach for dealing with clinical problems: question formulation, verification of latest evidence, critical appraisal and value assessment of evidence and clinical indication. Epidemiological studies have different weights on the evidence they provide. Additional information from in vivo studies, especially those that investigate the possible mechanism of alcohol toxicity to the oral mucosa, may complement the epidemiological evidence and provide possible benefits and risks for clinical use of alcohol containing mouthwashes. All of this information is then evaluated to ascertain whether the benefits of alcohol containing mouthwashes outweigh the risk of damage to the oral mucosa.

\section{QUESTION FORMULATION}

Do the clinical benefits of an alcohol containing mouthwash in the prevention of plaque related oral diseases outweigh the possible safety issues? The significant safety issue is developing oral squamous cell carcinoma. This raises the further question, is alcohol containing mouthwash a justifiable clinical treatment course to encourage patients to take?

\section{WEIGHTING EPIDEMIOLOGICAL EVIDENCE}

In an attempt to answer the questions set out above via an evidence-based approach, 
the epidemiological evidence needs to be quantified. The criteria for quantifying epidemiological evidence are shown in Table 1.

Neither of the review papers can be classified as systematic reviews as they have not included a randomised clinical trial (RCT) and do not follow strict reviewing protocols. Although this is a criticism, it should be identified that a RCT to show an association between oral cancer and alcohol containing mouthwash use would be difficult to carry out. Oral cancer is a chronic disease, can take many years to develop and an RCT will require a large follow-up population.

The possible risk association between alcohol containing mouthwashes and oral cancer is considered from five directly relevant case control studies (type IV evidence). In such studies, subjects were selected on the basis of whether they do (cases) or do not have a particular disease (controls). Information is then determined based on the calculated odds ratios (OR). It is important to differentiate here the fact that risk in another type of study (cohort study) is derived from risk ratios (RR), which are determined by a different mathematical formulation. The terms OR and RR have been used interchangeably in the literature evaluated and our assumption is that the calculation appropriate for case control studies was carried out, regardless of how it has been reported.

Some studies also refer to 'excess risk', which can introduce ambiguity in the terminology. Excess risk should be defined as the difference between the proportion of subjects in a population with oral cancer who were exposed to alcohol containing mouthwashes and the proportion of subjects with oral cancer who were not exposed. In the context of the literature on this subject, 'excess risk' seems to refer to statistically significant risk/odds ratio beyond 1.0.

\section{LATEST REVIEWS}

Two reviews have already been highlighted and these will be considered in further detail.

Review 1: 'The rate of alcohol in oral carcinogenesis with particular reference to alcohol containing mouthwashes. ${ }^{2}$

The review initially suggests an increased risk from alcohol containing mouthwashes in the development of oral cancer. This suggestion is based upon case control studies which examined the smoking-alcohol association in general, with no stratification of alcohol containing mouthwash use. ${ }^{4,5}$ The findings shown in a nation-based alcohol profile and oral cancer mortality correlation study failed to indicate that the weight of this association is not as strong as the one from case control studies. ${ }^{6}$

The main conclusion of this review is based upon the evidence provided by Guha et al., ${ }^{7}$ which uses data obtained from two multi-centred case control studies. Nevertheless, a particularly relevant finding from this study ${ }^{7}$ curiously was not mentioned, weakening the review further. The information related to self reported use of more than twice a day mouthwash indicated that it increased the chance of developing oral squamous cell carcinoma by almost six fold (OR 5.86; 95\% CI = 2.91, 11.77) when compared to those patients who reported never having used mouthwash products.

Lack of more in-depth explanation on existing evidence of association (or lack of association) gives the impression that the most conclusive epidemiological evidence should be derived solely from the most recent published study. The laboratory studies quoted in this review, at best, explore the link between alcohol and oral cancer as a facilitating factor rather than a causal or risk factor. A more balanced view on existing association between alcohol containing mouthwashes from case control studies would have increased the credibility of the conclusion from this paper. The review lacked depth of analysis on key issues, which brings into question the validity of its conclusions.

Review 2: 'Mouthwash and oral cancer risk: an update. ${ }^{3}$

This second review paper concluded that alcohol containing mouthwash use was not associated with an increased risk of oral squamous cell cancer. The author highlighted the lack of a dose response relationship. This review focused on the only two papers that specifically looked at alcohol containing mouthwashes as a risk for oral cancer. The studies were conducted by the US National Cancer Institute (NCI) and the findings of the first study ${ }^{8}$ were published in 1991. The second study ${ }^{9}$ was

\begin{tabular}{l|l}
\multicolumn{2}{l}{$\begin{array}{l}\text { Table } 1 \\
\text { epidemiological evidence }\end{array}$} \\
\hline Type I & $\begin{array}{l}\text { A least one good systematic review } \\
\text { (including at least one randomised } \\
\text { clinical trial) }\end{array}$ \\
\hline Type II & $\begin{array}{l}\text { At least one good randomised } \\
\text { clinical trial }\end{array}$ \\
\hline Type III & $\begin{array}{l}\text { Well designed interventional studies } \\
\text { without randomisation }\end{array}$ \\
\hline Type IV & $\begin{array}{l}\text { Well designed observational studies } \\
\text { (case control and cohort studies) }\end{array}$ \\
\hline Type V & $\begin{array}{l}\text { Descriptive studies (correlational } \\
\text { studies, cross sectional surveys), } \\
\text { case reports, case series, influential } \\
\text { reports and expert opinion }\end{array}$ \\
\hline
\end{tabular}

published in 2001 and a re-analysis of the 1991 data by authors not involved in the original study, followed in 2003. ${ }^{10}$

The 1991 study showed a statistically significant increase in risk of oral cancer associated with regular mouthwash use and suggested that the risk varied in proportion to dose, duration and frequency of mouthwash usage and alcohol concentration. The authors concluded that regular use of a mouthwash with high alcohol content contributes to oral cancer risk. The re-analysis of the same data ${ }^{10}$ divided the cases of oropharyngeal cancers into mucosal (true cases) and non-mucosal (pseudo cases). The re-analysis concluded that regular use of a mouthwash was associated more strongly with the pseudo cases than the true cases in women and there was a weak dose response relationship between mouthwash use and oropharyngeal cancer in pseudo cases. In men, the association between mouthwash use and true disease was confirmed, but considered weak evidence. The re-analysis also commented that such weak associations might reflect the use of mouthwashes by 'smokers' and 'drinkers' to conceal breath odours, as suggested by previous studies. ${ }^{11-14}$ One outcome of the 2003 re-analysis that was not commented upon was that women classified as 'true cases', those who had started to use mouthwashes before the age of 20 showed a significantly higher risk of oropharyngeal cancer as compared to controls $(\mathrm{RR}=2.3)$.

The 2001 NCI study reported no overall increased risk of oral cancer associated with mouthwash use.

The conclusion of the second review does not appear to provide a balanced view of the analysis of the data. The opposing 


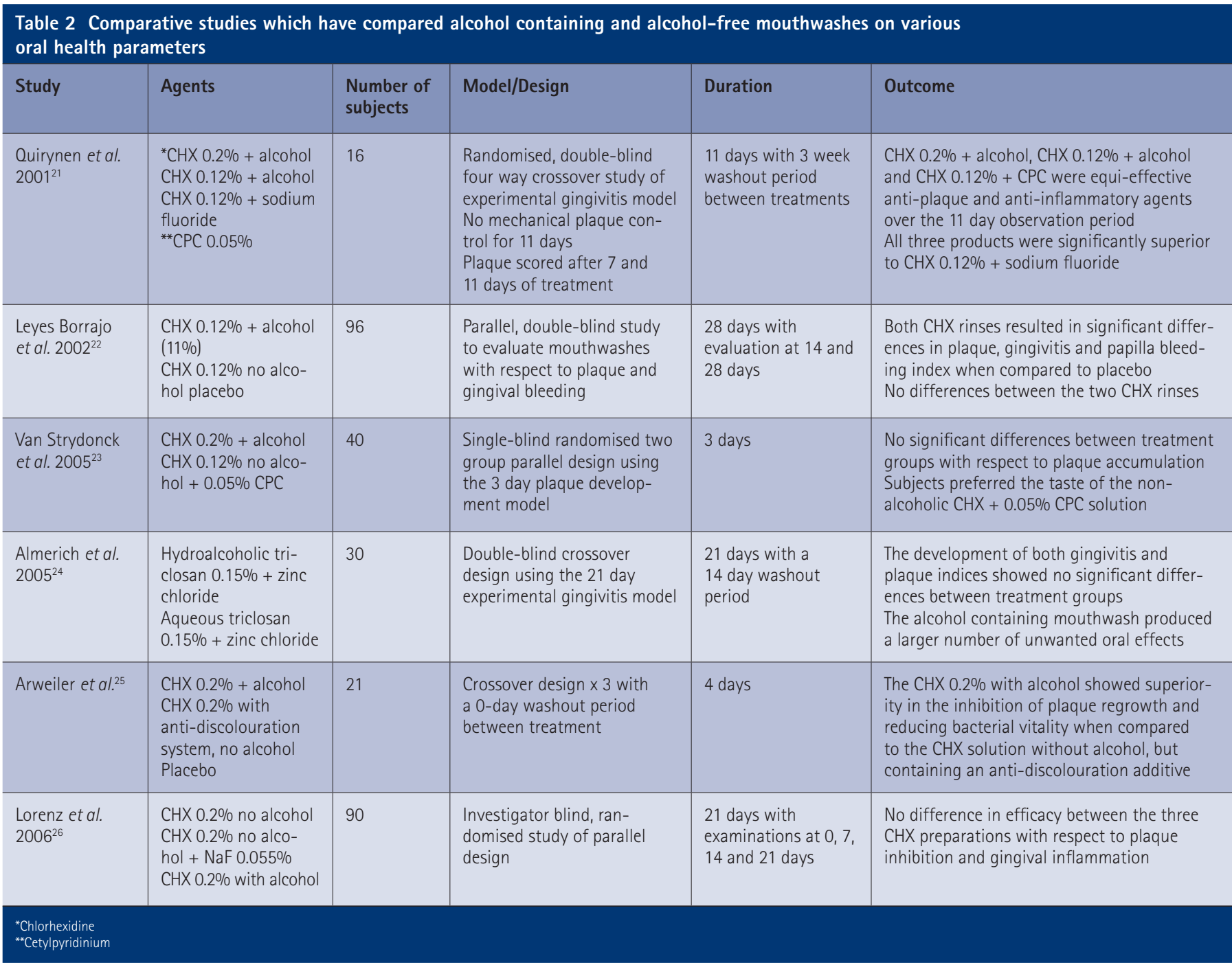

arguments were not considered. Results from previous case control studies ${ }^{12,14,15}$ were not mentioned and epidemiological evidence from case control studies showing no association between alcohol containing mouthwashes and oral cancer were scant.

\section{SAFETY AND EFFICACY OF ALCO- HOL CONTAINING MOUTHWASHES}

The incorporation of ethanol into mouthwashes serves several purposes: it is a solvent for other active ingredients, has antiseptic properties and acts as a preservative. Ethanol is easy to produce and relatively cheap.

In vitro studies have demonstrated that alcohol enhances the mucosal penetration of the various carcinogens found in tobacco. ${ }^{16}$ Alcohol on its own does cause damage to the oral mucosa and includes epithelial atrophy and decease in basal cell size atrophy with associated hyper-regeneration. ${ }^{17}$
The prime metabolite of alcohol is acetaldehyde, which is mutagenic and animal studies have shown this substance to be carcinogenic. ${ }^{18}$ While the bulk of the metabolism of alcohol is carried out in the liver, there is evidence that alcohol metabolism could occur in the oral cavity and that various bacteria in plaque can metabolise alcohol to acetaldehyde. This may support the only theory for why patients with poor oral hygiene are at an increased risk of oral cancer.

In addition to the possible risk of oral cancer, alcohol containing mouthwashes are also reported to have other adverse effects on oral structures and functions. These include burning mouth, drying of the oral mucosa, softening effects on composite filling materials and mucosal pain. ${ }^{19,20}$

The concern over the alcohol content of mouthwashes has led to the development of alcohol-free preparations. Various studies have been completed comparing the active mouthwash ingredient incorporated into an alcohol-free preparation with alcohol mouthwash, but a full systematic review has yet to be carried out. However, relevant studies obtained via a Medline search are listed in Table 2 and show that alcohol containing mouthwashes afford little or no advantage in terms of efficacy over the alcohol-free competitors.

\section{CONCLUSION}

Two review papers ${ }^{2,3}$ have reviewed the evidence suggesting alcohol containing mouthwashes as a risk for the development of oral cancer and also cancer of the mucosal surfaces of the upper aerodigestive tract. The conclusion from these two reviews is conflicting. Both papers have been selective in the studies that they have reviewed on this topic and their interpretation of the data. There is evidence to show an association between use of an alcohol containing mouthwash and oral cancer. However, this evidence 
is weak and inconclusive. A robust randomised controlled trial would be necessary to answer the question of whether alcohol containing mouthwashes increase the risk of developing oral cancer. The feasibility and justification for such a trial is likely to raise ethical and logistical questions.

Against this background there is overwhelming evidence that mouthwashes are of value in reducing bacterial plaque and gingivitis and are useful adjuncts to mechanical methods of plaque removal. The comparative studies between alcohol containing and alcohol-free mouthwashes have for the most part shown that the alcohol content adds little in the way of efficacy to the product. Besides this, alcohol containing mouthwashes may be also avoided on the basis of cultural preferences. Therefore, as the benefit of alcohol in a mouthwash is negligible and it may carry a risk of oral cancer which is difficult to quantify, is there any value in members of the dental team prescribing/ recommending alcohol containing mouthwashes to their patients?

1. Lachenmeier D W. Safety evaluation of topical applications of ethanol on the skin and inside the oral cavity. J Occup Med Toxicol 2008; 3: 26

2. McCullough M J, Farah C S. The role of alcohol in oral carcinogenesis with particular reference to alcohol containing mouthwashes. Aust Dent J 2008;
53: 302-305.

3. La Vecchia C. Mouthwash and oral cancer risk: an update. Oral Oncol 2009; 45: 198-200.

4. Castellsagué $\mathrm{X}$, Quintana M J, Martínez M C et al. The role of type of tobacco and type of alcoholic beverage in oral carcinogenesis. Int J Cancer 2004; 108: 741-749

5. Maserejian N N, Joshipura K J, Rosner B A Giovannucci E, Zavaras A I. Prospective study of alcohol consumption and risk of oral pre malignant lesions in men. Cancer Epidemiol Biomarkers Prev 2006; 15: 774-778.

6. Petri S, Scully C. Oral cancer: the association between nation-based alcohol drinking profiles and oral cancer mortality. Oral Oncol 2005; 41: 828-834.

7. Guha N, Boffetta P, Wünsch Filho V et al. Oral health and risk of squamous cell carcinoma of the head and neck and esophagus: results of two multicentric case control studies. Am J Epidemiol 2007: 116: 1159-1173.

8. Winn D M, Blot W J, McLaughlin J K et al. Mouthwash use and oral conditions in the risk of oral and pharyngeal cancer. Cancer Res 1991; 51: 3044-3047.

9. Winn D M, Diehl S R, Brown L M et al. Mouthwash in the aetiology of oral cancer in Puerto Rico. Cancer Causes Control 2001; 12: 419-429.

10. Cole P, Rodu B, Mathisen A. Alcohol containing mouthwash and oropharyngeal cancer: a review of the epidemiology. J Am Dent Assoc 2003; 134: 1079-1087.

11. Wynder E L, Kabat G, Rosenberg S, Levenstein M. Oral cancer and mouthwash use. J Natl Cancer Inst 1983; 70: 255-260.

12. Mashberg A, Barsa P, Grossman M L. A study of the relationship between mouthwash use and oral and pharyngeal cancer. J Am Dent Assoc 1985; 110: 731-734.

13. Young T B, Ford C N, Brandenburg J H. An epidemiologic study of oral cancer in a statewide network. Am J Otolaryngol 1986; 7: 200-208

14. Kabat G C. Hebert J R, Wynder E L. Risk factors for oral cancer in women. Cancer Res 1989; 49: 2803-2806

15. Blot W J, Winn D M, Fraumeni J F Jr. Oral cancer and mouthwash. J Nat/ Cancer Inst 1983: 70: 251-253.

16. Squier C A, Cox P, Hall B K. Enhanced penetration of nitroso-nornicotine across oral mucosa in the presence of ethanol. J Oral Pathol 1986; 15: 276-279.

17. Howie N M, Trigkas T K, Cruchley A T, Wertz P W, Squier C A, Williams D M. Short term exposure to alcohol increases the permeability of human oral mucosa. Oral Dis 2001; 7: 340-354.

18. WHO International Agency for Research on Cancer. Re-evaluation of some organic chemicals, hydrazine and hydrogen peroxide. IARC Monographs on the Evaluation of Carcinogenic Risks to Humans, vol. 71. Part 1: pp 1-315. Lyon: IARC, 1999.

19. Settembrini $L$, Penugonda B, Scherer W, Strassler $H_{\text {, }}$ Hittelman E. Alcohol containing mouthwashes: effects on composite colour. Oper Dent 1995; 20: 14-17.

20. Weiner R, Millstein P, Hoang E, Marshall D. The effect of alcoholic and non-alcoholic mouthwashes on heat-treated composite resins. Oper Dent 1997; 22: 249-253.

21. Quirynen $M$, Avontroodt $P$, Peeters $W$, Pauwels $M$ Coucke W, Van Steenberghe D. Effect of different chlorhexidine formulations in mouthrinses on de novo plaque formation. J Clin Periodontol 2001; 28: 1127-1136.

22. Leyes Borrajo J L, Garcia V L, Lopez C G, RodriguezNuñes I, Garcia F M, Gallas T M. Efficacy of chlorhexidine mouthrinses with and without alcohol: a clinical study. J Periodonto/ 2002; 73: 317-321.

23. Van Strydonck D A C, Timmerman M F, van der Velden U, van der Weijden G A. Plaque inhibition of two commercially available chlorhexidine mouthrinses. J Clin Periodontol 2005; 32: 305-309.

24. Almerich J M, Canedo N, Ortolá J C, Poblet J. Influence of alcohol in mouthwashes containing triclosan and zinc: an experimental gingivitis study. IClin Periodontol 2005; 32: 539-544.

25. Arweiler N B, Boehnke N, Sculean A, Hellwig E, Auschill T M. Differences in efficacy of two commercial 0.2\% chlorhexidine mouthrinse solutions: a 4-day plaque re-growth study. J Clin Periodontol 2006; 33: 334-339.

26. Lorenz K, Bruhn G, Heumann C, Netuschil L, Brecx M, Hoffmann T. Effect of two new chlorhexidine mouthrinses on the development of dental plaque, gingivitis and discolouration. A randomised, investigator-blind, placebo-controlled 3 week experimental gingivitis study. J Clin Periodontol 2006; 33: 561-567. 